Development 140, 3303-3309 (2013) doi:10.1242/dev.093096 (C) 2013. Published by The Company of Biologists Ltd

\title{
Adult neural stem cells: plastic or restricted neuronal fates?
}

\author{
Eduardo B. Sequerra ${ }^{1, *}$, Marcos R. Costa², João R. L. Menezes ${ }^{3,4}$ and Cecilia Hedin-Pereira ${ }^{4,5}$
}

\begin{abstract}
Summary
During embryonic development, the telencephalon is specified along its axis through morphogenetic gradients, leading to the positional-dependent generation of multiple neuronal types. After embryogenesis, however, the fate of neuronal progenitors becomes more restricted, and they generate only a subset of neurons. Here, we review studies of postnatal and adult neurogenesis, challenging the notion that fixed genetic programs restrict neuronal fate. We hypothesize that the adult brain maintains plastic neural stem cells that are capable of responding to changes in environmental cues and generating diverse neuronal types. Thus, the limited diversity of neurons generated under normal conditions must be actively maintained by the adult milieu.
\end{abstract}

Key words: Adult neurogenesis, Cerebral cortex, Neuronal specification, Olfactory bulb, Subventricular zone

\section{Introduction}

In the developing telencephalon, the localized release of morphogens generates gradients that confer information related to the spatial coordinates and positioning of cells (positional information) (Gunhaga et al., 2000; Gunhaga et al., 2003). Such morphogen gradients induce the differential expression of transcription factors (TFs) along the dorsoventral axis, creating segregated germinal zones (Schuurmans and Guillemot, 2002). For example, neural progenitors within the mouse dorsal telencephalon, identified as radial glial cells (RGCs; Kriegstein and AlvarezBuylla, 2009), express the TFs neurogenin 2 (Neurog2) and empty spiracles homologue 1 (Emx1), and generate glutamatergic neurons (Gorski et al., 2002; Schuurmans et al., 2004). By contrast, RGCs located in the ventral telencephalon express different sets of TFs, such as distal-less homeobox 1 (Dlx1) and Dlx2, NK2 homeobox 1 (Nkx2.1), LIM homeobox protein 6 (Lhx6), GS homeobox 2 (Gsh2; Gsx2 - Mouse Genome Informatics) or Nkx6.2, and contribute GABAergic neurons to the cortex (Anderson et al., 1997; Fogarty et al., 2007; Xu et al., 2008). In primates, this dorsoventral separation in the origin of glutamatergic and GABAergic neurons may be less strict, and some mixture between these progenitors has been suggested in the dorsal ventricular zone (VZ; Letinic et al., 2002; Jakovcevski et al., 2011).

The accretion of neurons from distinct germinal zones is also a feature of the olfactory bulb (OB), the most anterior vertebrate telencephalic structure. In rodents, the two main types of

\footnotetext{
${ }^{1}$ Department of Physiology and Membrane Biology, University of California Davis, Shriners Hospital for Children Northern California, 2425 Stockton Boulevard, Sacramento, CA 95817, USA. ${ }^{2}$ Brain Institute, Federal University of Rio Grande do Norte, Rio Grande do Norte, RN 59078-900, Brazil. ${ }^{3}$ Programa de Anatomia, Laboratório de Neuroanatomia Celular, Instituto de Ciências Biomédicas, Universidade Federal do Rio de Janeiro, Rio de Janeiro, RJ 21941-590, Brazil. ${ }^{4}$ Universidade Federal do Rio de Janeiro, Rio de Janeiro, RJ 21941-590, Brazil. 5 Instituto de Biofísica Carlos Chagas Filho, Rio de Janeiro, RJ 21941-590, Brazil.

*Author for correspondence (ebsequerra@ucdavis.edu)
}

glutamatergic projection neurons in the $\mathrm{OB}$ - mitral neurons and tufted neurons - are generated exclusively during embryonic stages (Hinds, 1968a; Hinds, 1968b; Bayer, 1983) from dorsal progenitors expressing the TFs Emx1 (Gorski et al., 2002) and Neurog2 (Winpenny et al., 2011). By sharp contrast, local circuit neurons, which are mostly GABAergic neurons that can also express dopamine (Kosaka et al., 1985; Betarbet et al., 1996; Hack et al., 2005), start to be generated embryonically by ventrolateral progenitors expressing the TF Er81 (ets variant gene 1; Etv1 Mouse Genome Informatics) (Stenman et al., 2003), and are continuously generated throughout life by progenitors located within the whole extension of the postnatal and adult subventricular zone (SVZ) (Altman, 1969; Bayer, 1985; Luskin, 1993; BatistaBrito et al., 2008). Fate-mapping experiments using the Cre-Lox system have provided evidence that these progenitors in the postnatal SVZ are derived from distinct germinative niches of the developing telencephalon (Kohwi et al., 2007; Young et al., 2007). Intriguingly, these experiments have shown that a large fraction of postnatal SVZ progenitors engaged in the generation of $\mathrm{OB}$ GABAergic neurons are actually derived from dorsal, Emx1expressing territories in the developing telencephalon, where only glutamatergic neurons are generated during embryogenesis (Gorski et al., 2002). The contribution of these dorsal progenitors to the adult SVZ neuronal progenitor pool was confirmed by the specific labelling of their radial extension to the dorsal pia mater (Ventura and Goldman, 2007; Merkle et al., 2007; Kelsch et al., 2007). These studies highlighted that these cells can give rise to adult SVZ neural stem cells (NSCs) that maintain their position and to neurons that migrate to the $\mathrm{OB}$ and differentiate into GABAergic interneurons. Collectively, these data suggest that the same progenitor cell population that generates glutamatergic neurons during embryonic development can also generate GABAergic neurons during postnatal stages (Fig. 1), although we cannot completely rule out the possibility that postnatal/adult NSCs are derived from a subpopulation of elusive RGCs that remain quiescent and do not give rise to glutamatergic cortical neurons during embryogenesis (Costa et al., 2010).

Given the high contribution of Emx1-derived progenitors to the postnatal/adult SVZ (Kohwi et al., 2007; Young et al., 2007), and assuming that Emx1-derived postnatal/adult NSCs belong to the lineage of multipotent RGCs that generated glutamatergic neurons during embryogenesis (Costa et al., 2010), it thus appears that postnatal SVZ NSCs exhibit a reduced capacity to generate diverse neuronal types in comparison with their embryonic counterparts. This restriction in the phenotypic repertoire of cells generated by postnatal NSCs could be attributed to genetic programs inherited by SVZ progenitors at early developmental stages (Merkle et al., 2007). However, several lines of evidence suggest that the fate of postnatal/adult NSCs can be strongly influenced by environmental changes. In this Hypothesis, we review studies that have addressed the plasticity of neural stem cells and progenitors, and we hypothesize that SVZ progenitors retain the potential to generate a much larger diversity of neuronal subtypes, but are constrained by a continuous feeding of restrictive signals. 


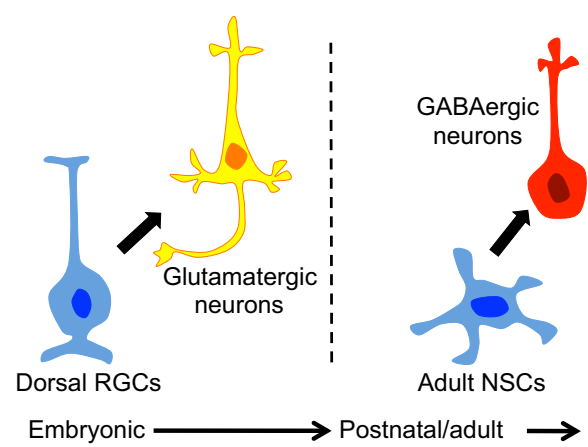

Fig. 1. Neuronal types generated by telencephalic neural stem cells. Dorsal embryonic neural stem cells (NSCs), also known as radial glial cells (RGCs, blue), generate cortical glutamatergic pyramidal neurons (yellow), either directly or via transit amplifying progenitors (not shown). After embryogenesis, RGCs give rise to adult NSCs (blue), which are subventricular zone astrocytes that maintain their dorsal position. These cells and their progeny start giving rise to a completely different neuronal type: GABAergic olfactory bulb interneurons (red). The dashed line represents the transition between embryonic and postnatal neurogenesis.

\section{Environmental signals and neuronal plasticity in the developing CNS}

Neuronal subtype specification has been largely studied in the vertebrate spinal cord, a structure that is amenable to experimental manipulations. Similar to the telencephalon, progenitor specification in the developing spinal cord is defined by the action of morphogenetic gradients. In this structure, bone morphogenetic protein $4(\mathrm{Bmp} 4)$ released by the overlying ectoderm opposes sonic hedgehog (Shh) and creates morphogenetic territories that determine the differential expression of TFs and, consequently, the differentiation of neuronal subtypes according to their dorsoventral position (Jessell, 2000). However, this TF expression profile induced by early morphogenetic gradients does not seem to be sufficient to sustain a thorough differentiation program (Dessaud et al., 2010; Gabay et al., 2003).

Dessaud et al. (Dessaud et al., 2010) have shown that neural progenitors in chick neural tube explants are induced to follow specific neuronal lineages (V0, V1, V2 and motoneurons) according to the surrounding Shh concentration. However, if Shh signalling is interrupted, the progeny shift to more dorsal phenotypes. At later stages, when the TF domains are already established, the genetic and pharmacological inhibition of Shh signalling in vivo still leads to a shift towards dorsal phenotypes (Dessaud et al., 2010). These results, together with earlier work (Gabay et al., 2003), indicate that the apparent determination of progenitors by specific transcription factors may not be a final event and that progenitor fate can be redirected by environmental cues.

Studies have shown that Shh signalling also interferes with the specification of different GABAergic neuron subtypes in the ventral telencephalon (Xu et al., 2005; Xu et al., 2010). As Shh signalling is important for the early regionalization of the telencephalic vesicle (Gunhaga et al., 2000), these studies used conditional knockout techniques to abolish Shh signalling in progenitor cells after telencephalon patterning had taken place. By doing this, the authors provided evidence that Shh signalling is important to maintain the fate of ventral telencephalic progenitors and control the generation of different subtypes of cortical GABAergic neurons (Xu et al., 2005; Xu et al., 2010). Therefore, a continuous feeding of positional information is also required in the telencephalon for progenitors to differentiate into the appropriate neuronal type.

Further evidence for the crucial influence of environmental signals on neuronal specification in the telencephalon comes from in vitro studies. Pioneering experiments have shown that cultured embryonic cerebral cortex progenitor cells can produce catecholaminergic neurotransmitters (Iacovitti et al., 1987), which are not normally expressed in vivo. Furthermore, Götz and Bolz (Götz and Bolz, 1994) have shown that proliferating progenitors dissociated from embryonic cortical tissue, fail to express GABA or glutamate after 1 week in culture (in contrast with progenitors that left the cell cycle 1 or 3 days prior to dissociation). This suggests that, even after acquiring positional information, postmitotic neurons require environmental instruction for their neurotransmitter choice. Likewise, E13 dorsal telencephalic progenitors that in situ do not generate GABAergic neurons are able to do so in vitro, albeit at low numbers (He et al., 2001). Additionally, progenitors from the E11.5 dorsolateral telencephalon produce more interneurons than their dorsomedial counterparts after 7 days in vitro (Gulacsi and Lillien, 2003). The number of GABAergic neurons generated by dorsomedial progenitors can be increased if they are co-cultured with ventral cells, suggesting the presence of inductive signals. In fact, this induction is inhibited by addition of the Shh antagonist cyclopamine and enhanced by exogenous Shh addition. An opposite effect is observed with Bmp4, which reduces the number of GABAergic cells generated (Gulacsi and Lillien, 2003).

The migration of progenitors from ventral to dorsal germinative zones (Fishell et al., 1993), as well as in the dorsal to ventral direction (Willaime-Morawek et al., 2006; Cocas et al., 2009), could also contribute to the switch in the generation of neuronal subtypes in the embryonic telencephalon. Cocas et al. (Cocas et al., 2009), for example, showed that Emx1-derived cells migrating from dorsal to ventral telencephalon contribute inhibitory medium spiny neurons in the striatum. Therefore, neurotransmitter specification in the embryonic telencephalon seems to be a sum of early positional, intrinsic, information and the extrinsic signals coming from the actual environment.

Electrical activity can also interfere with neuronal specification. During development, cells in the neural tube assume different electric activity behaviours according to their position along the dorsoventral axis (Borodinsky et al., 2004). Cells in the ventral neural tube display a higher frequency of calcium spikes than those in the dorsal region. Through genetic and pharmacological manipulation of spike frequency, Borodinsky et al. (Borodinsky et al., 2004) have shown that cells exhibiting increased calcium spikes choose to express the inhibitory neurotransmitters GABA and glycine, whereas decreased calcium spikes lead to the expression of glutamatergic and cholinergic phenotypes.

These fate changes imply an overwriting or suppression of genetic programs previously established. In fact, in the spinal cord, the TFs T-cell leukaemia 1 (Tlx1) and Tlx 3 determine the choice between glutamatergic and GABAergic phenotypes (Cheng et al., 2004). By suppressing calcium transients, which increase the generation of vesicular glutamate transporter-immunoreactive cells, and inhibiting Tlx3 expression simultaneously, Marek et al. (Marek et al., 2010) observed that the phenotype produced was similar to a simple Tlx3 knockdown: an increase in GABAergic neuron fate. Similarly, decreasing calcium transients while overexpressing Tlx3 caused an increase in the number of glutamatergic cells (Marek et al., 2010). These data show that the influence of electrical activity over neurotransmitter choice acts upstream of the genetic program 
encoded by TF expression. Indeed, the Tlx3 gene has a cAMP response element site that is recognized by Jun. Jun phosphorylation is activity dependent and is the link between activity and the genetic trait that leads to glutamatergic fate in the spinal cord (Marek et al., 2010).

A recent study showed that Shh signalling also enhances calcium spikes in the embryonic neural tube, through activation of its coreceptor smoothened (Belgacem and Borodinsky, 2011). In this study, the authors reported that cells at the ventricle surface do not display spontaneous activity, whereas those on the outer surface are the ones that display activity-dependent neurotransmitter choice. As the outer surface is mainly composed of postmitotic neurons, these data suggest that postmitotic neurons are still responsive to Shh. Thus, Shh signalling regulates the pattern of spontaneous activity by these cells that is instructive for neurotransmitter specification.

In summary, our view of neurotransmitter specification in the developing CNS, which was previously thought to be an early event determined by positional information and immutable TF codes, is changing towards a model in which determination is the sum of a series of changeable events.

\section{Modifications to the micro-environment and neuronal plasticity in the postnatal/adult CNS}

If the ideas discussed above could be transposed to the postnatal/adult CNS, we would expect that SVZ progenitors, instead of being simple followers of early determinants, would be plastic and capable of changing their potential. This was the main view following early adult NSC transplantation studies in which hippocampal NSCs generated OB neurons that express dopamine (Suhonen et al., 1996), a neurotransmitter that they do not express when they remain in the hippocampus. Thus, the putative genetic program that restricts postnatal SVZ NSCs to generate mostly GABAergic neurons would be under constant control by environmental signals, which could in particular cases reprogram these NSCs towards the generation of distinct neuronal subtypes, such as pyramidal glutamatergic neurons.

In line with this idea, several groups have recently reported lesion-induced addition of new projection/excitatory neurons to the cerebral cortex after birth (Magavi et al., 2000; Chen et al., 2004; Fagel et al., 2009; Vessal and Darian-Smith, 2010; Bi et al., 2011). However, this generation of new neurons in the postnatal/adult cortex seems to be dependent on unknown signals: those not usually elicited by most types of lesions (Saha et al., 2012; Diaz et al., 2013). It is also still unclear whether these new neurons could be derived from progenitor cells in the parenchyma itself (Palmer et al., 1999; Guo et al., 2010) or the marginal zone/layer 1 (Costa et al., 2007; Ohira et al., 2010).

There is also other strong evidence to support the notion that excitatory neurons could be derived from the postnatal/adult SVZ. Using co-cultures of postnatal and adult SVZ explants with embryonic telencephalic slices, one study (Sequerra et al., 2010) has shown that SVZ cells migrate into the cortical plate and differentiate into different neuronal types, including glutamatepositive neurons with pyramidal morphology. These observations indicate that the postnatal/adult SVZ harbours progenitors that are capable of generating glutamatergic neurons, even though this differentiation seems to be hampered under physiological conditions in the adult mammalian brain (Fig. 2). In addition, Brill and colleagues have provided evidence that dorsal SVZ progenitors are not completely blocked in their capacity to generate glutamatergic neurons in vivo (Brill et al., 2009). They
A Physiological conditions

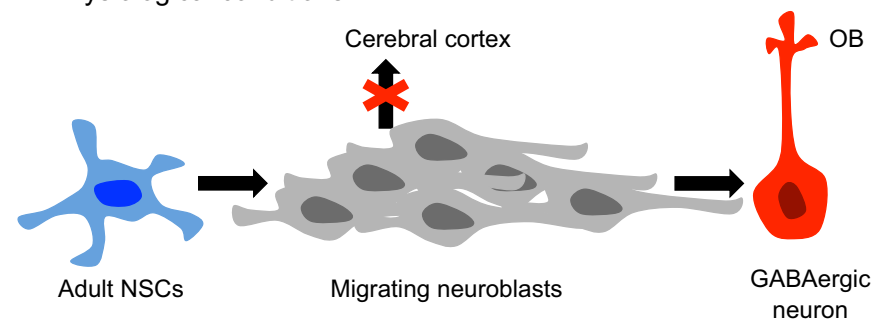

B Homeostasis disruption conditions

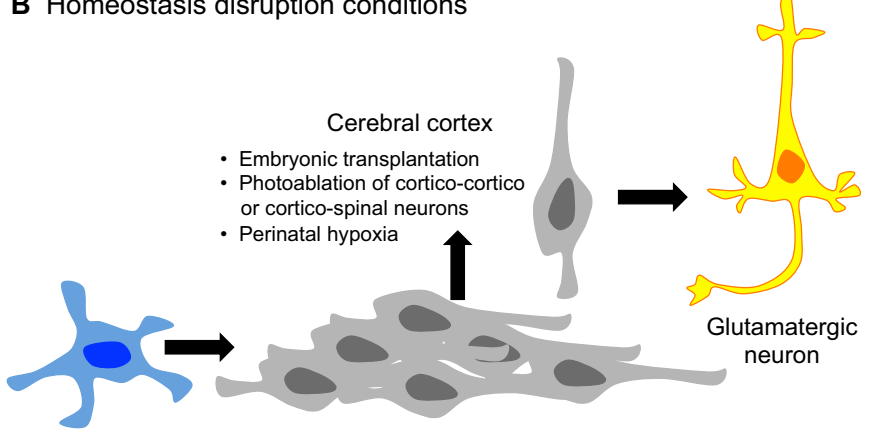

Fig. 2. Environmental regulation of subventricular zone (SVZ) neuronal progenitor fate. (A) Under physiological conditions, postnatal neuronal precursors (grey) generated in the SVZ migrate to the olfactory bulb $(O B)$ and give rise to GABAergic $O B$ neurons (red). As they migrate, they are inhibited from invading the surrounding parenchyma along their migratory route. Our hypothesis predicts that signals presented to them lead to the differentiation of GABAergic inhibitory local circuitry neurons in this structure. (B) When homeostasis is disrupted (via the listed possibilities), SVZ neuroblasts are stimulated to invade the parenchyma and to adopt different fates, such as the glutamatergic pyramidal cortical neuron.

demonstrated that the adult SVZ contains a population of progenitors identified by a set of TFs commonly found in the dorsal embryonic VZ, such as Neurog2, Tbr1 and Tbr2 (Eomes - Mouse Genome Informatics). These progenitors are located in the dorsal aspect of the adult SVZ and give rise to a class of glutamatergic OB interneurons known as 'short axon cells' (Brill et al., 2009). This was the first description of postnatal glutamatergic neurons generated in the uninjured adult SVZ.

Collectively, these studies (Brill et al., 2009; Sequerra et al., 2010) clearly demonstrate the potential of postnatal and adult SVZ progenitor cells to generate glutamatergic neurons either under physiological or induced conditions. However, several important issues remain. For example, why do most adult SVZ progenitors derived from dorsal telencephalic radial glia generate GABAergic cells (Young et al., 2007; Ventura and Goldman, 2007; Merkle et al., 2007)? Do these SVZ progenitors indeed belong to the same lineage of dorsal radial glia that generated cortical glutamatergic neurons during embryogenesis? If so, does this change in fate commitment rely on cell-intrinsic or -extrinsic mechanisms, or both?

\section{SVZ progenitor diversity and commitment}

New neurons generated by the SVZ arise from a lineage composed of NSCs that rarely proliferate and survive mitotic ablation following treatment with the anti-mitotic agent arabinofuranosyl cytidine (AraC) (Doetsch et al., 1999). These slow-dividing NSCs give rise to fast-dividing astrocytic B cells (Costa et al., 2011) and 
intermediate progenitors (type $\mathrm{C}$ cells) that amplify the progenitor pool (Doetsch et al., 1997; Costa et al., 2011). Intermediate progenitors give rise to neuroblasts (A cells) (Doetsch et al., 1997) that display high mobility and are able to proliferate while migrating (Menezes et al., 1995; Lois et al., 1996). Our interest here is to understand when and how these various precursors become committed to a specific neuronal type and whether this commitment can be reversed or modified.

Interestingly, among the TFs involved in neuronal phenotype specification in the SVZ, none is expressed in astrocytes. Pax6, a determinant of dopaminergic neurons, is expressed mostly by neuroblasts, mainly when they are closer to the dorsal aspect of the lateral ventricle (Hack et al., 2005; Brill et al., 2008). Dlx2, which in combination with Pax6 promotes dopaminergic periglomerular phenotype (Brill et al., 2008), starts to be expressed in type C cells and later in neuroblasts (Doetsch et al., 2002). The transcription factors involved in glutamatergic short-axon cell generation, such as Neurog2, Tbr2 and Tbr1, are expressed only in neuroblasts (Brill et al., 2009). Therefore, although the different neuronal types are generated in a regionally segregated manner, the idea that this commitment is inherited at the NSC phase is not sustained by the pattern of TFs expressed in the various progenitors (Fig. 3). Notably, Beckervordersandforth et al. (Beckervordersandforth et al., 2010) have shown that although neuronal subtype-specific TFs are not expressed by SVZ NSCs, the mRNAs for these molecules are enriched in this population. This early expression of neurogenic TF mRNA has been interpreted as neurogenic priming, and probably reflects an early step of differentiation that precedes any phenotypic commitment. Indeed, several lines of evidence demonstrate that neuronal phenotype may still be altered in the lineage of SVZ cells after the NSC stage (Hack et al., 2005).

Paradoxically, other data suggest an early commitment of neuronal phenotype. Merkle et al. (Merkle et al., 2007) have shown that the different $\mathrm{OB}$ populations in the adult are generated in a spatially segregated manner along the lateral ventricle dorsoventral axis: dorsal progenitors give rise to periglomerular layer $\mathrm{TH}^{+}$cells, whereas ventral progenitors give rise to glomerular calbindin ${ }^{+}$cells (Merkle et al., 2007). The same segregation occurs in the granular layer, where dorsal progenitors generate preferentially superficial

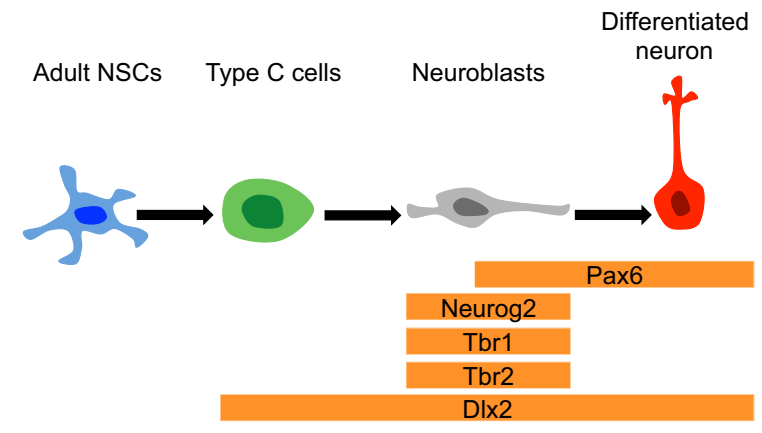

Fig. 3. Pattern of neuronal type transcription factor (TF) expression in subventricular zone (SVZ) cells. Adult neural stem cells (NSCs; SVZ astrocytes or type B cells, blue) do not express any of the described neuronal type-specific TFs (orange). These astrocytes give rise to transit amplifying precursors (type $C$ cells, green) that express DIx2. Type $C$ cells then generate migratory neuroblasts (type A cells, grey) of which a subpopulation transiently expresses Neurog2, Tbr1 and Tbr2. Pax6 is also expressed by neuroblasts and, as for Dl×2, its expression is sustained even after differentiation into OB interneurons (red). Dlx2, distal-less homeobox 2; Neurog2, neurogenin 2; Pax6, paired box gene 6; Tbr1, T-box brain gene; Tbr2, eomesodermin homologue. granular neurons and ventral progenitors generate deep granular neurons (Merkle et al., 2007). These populations display different patterns of connectivity. Superficial granular neurons project dendrites to the external plexiform layer where they contact tufted cells (Orona et al., 1983; Mori et al., 1983), and deep granular neurons project their dendrites to contact mitral neurons (see also Shepherd, 1990). Using an approach similar to Merkle et al. (Merkle et al., 2007), Kelsch et al. (Kelsch et al., 2007) transplanted SVZ explants labelled with retrovirus heterotopically and showed that the granular neurons displayed position and dendritic pattern according to their site of origin, although it should be noted that the cellular environment was also transplanted in this study. Furthermore, Merkle et al. (Merkle et al., 2007) have shown that neurospheres derived from different regions of the SVZ when transplanted to heterotopic positions give rise to $\mathrm{OB}$ neurons according to their origin, again suggesting that neurosphere-forming cells of the SVZ are already instructed before transplantation.

However, in support of our hypothesis, the same group showed recently that Shh signalling is continuously active in the ventral $\mathrm{SVZ}$ and that this signalling is important for the specification of the ventral progenitor subpopulation (Ihrie et al., 2011). Disruption of Shh signalling in vivo dorsalizes ventral progenitors (i.e. progenitors that normally give rise to superficial granular cells), whereas the expression of a constitutively active Shh receptor, Smo, in the dorsal SVZ leads to the generation of ventralized neurons (Ihrie et al., 2011). These data show that SVZ ventral progenitors are plastic and depend on Shh signalling to acquire their final phenotype. It also highlights that positional information can be instructive (regarding the expression of Shh receptors) but not determinant of the final phenotype.

Another example of phenotypic change based on environmental factors comes from experiments showing that the extracellular infusion of GABA into OB neurons causes them to depolarize and increase the expression of GFP driven by the tyrosine hydroxylase promoter, indicating dopaminergic neuron differentiation (Akiba et al., 2009). These data collectively indicate that neuronal specification of SVZ progenitors can be modulated by extrinsic factors, namely electrical activity and morphogens (Fig. 4).

It is not clear why the transplanted cells in the experiments described by Merkle et al. (Merkle et al., 2007) did not seem to respond to the local environment. One possibility could be that transplanted cells did not assimilate the local signalling [as discussed by Ihrie et al. (Ihrie et al., 2011)]. In fact, the authors first transplanted dissociated cells from different regions containing retroviral-infected RGCs. These transplanted infected cells behaved typically like donor tissue, but the authors could not rule out the possibility that unlabelled cells grafted alongside the labelled cells could be carrying factors from the donor environment. For this reason, they then adopted the neurosphere assay, in which cells were passaged three times before transplanting. In this way, RGCs (and their offspring) could be isolated from any environmental factors in the donor tissue. An alternative interpretation in this case however, is that the neurosphere assay would instead select specified cells. It is known that neurosphere-forming cells are proliferating cells that express EGFR, which is typically present in fast dividing type B and $\mathrm{C}$ cells (for a review, see Pastrana et al., 2011). Although both proliferative and quiescent cells in the SVZ contribute to neurosphere-forming potential (Doetsch et al., 2002; Imura et al., 2003; Morshead et al., 2003; Garcia et al., 2004), it is likely that the faster proliferating and more numerous type $\mathrm{C}$ cells predominate in these assays (Doetsch et al., 2002; Pastrana et al., 2009). For these reasons, we propose that neuronal type specification begins in cells 


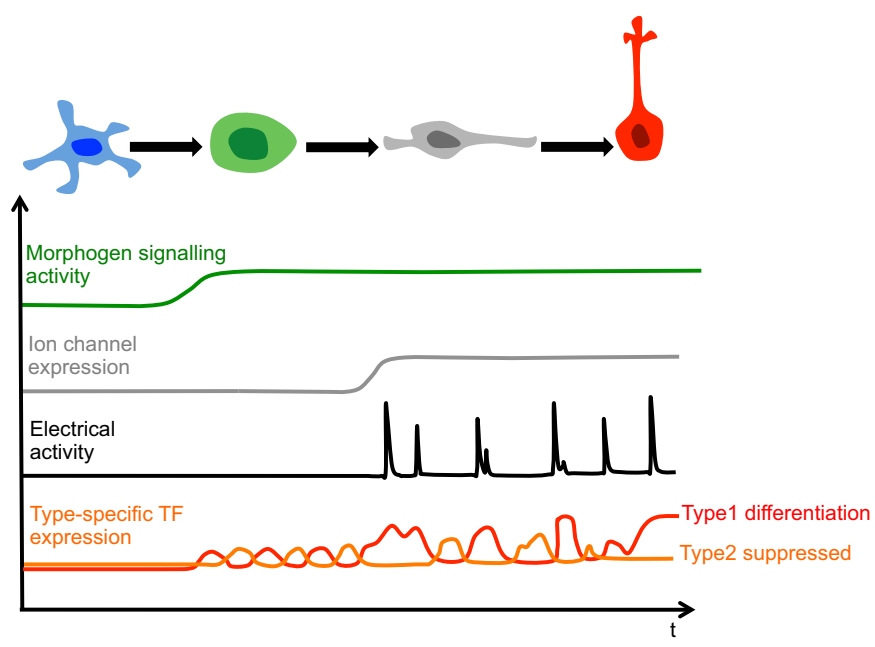

Fig. 4. Model for interaction of morphogen signalling, electrical activity and the expression of type-specific transcription factor (TFs) during subventricular zone (SVZ) neurogenesis. Neurogenesis by SVZ progenitors follows a four-step sequence consisting of the neural stem cell stage (type $B$, blue), the amplifying fast dividing progenitor stage (type $C$, green), the fast dividing neuroblasts (type A, grey) and the post-mitotic neurons (red). Extrinsic signalling molecules (i.e.

neurotransmitters, morphogens) are present in the SVZ milieu, but the sensitivity of cells to this signalling varies with their receptor expression pattern [e.g. epidermal growth factor (EGF) receptors are expressed by $C$ cells; $N$-methyl-D-aspartate (NMDA) receptors are expressed only in neuroblasts]. The model predicts the existence of a neural stem cell that remains mostly unresponsive to these external signalling molecules. During the neuroblast phase, cells start displaying electrical activity. Both morphogen signalling and electrical activity regulate the expression of type-specific TFs. We predict that the pattern of expression of the TFs is oscillatory and that the same cell may oscillate the expression of more than one TF until stabilization occurs, which leads to different neuronal subtypes being specified. There is evidence for persistent signalling by sonic hedgehog (Shh) and bone morphogenetic protein (BMP), although morphogens may or may not persist at a constant level as depicted. The curves are simply illustrative.

that are in between the type $\mathrm{C} /$ neuroblast transition and that there is a reservoir of uncommitted stem cells that generates them. This idea could be initially tested by heterotopic transplantations of hGFAP$\mathrm{GFP}^{+}$/prominin $1^{+}$cells, which are slow-dividing $\mathrm{B}$ cells (Beckervordersandforth et al., 2010).

It is still not clear when the window for commitment closes, if ever, as changes in environmental factors can induce different phenotypic choices after type-specific TFs start being expressed. As discussed above, in the developing ventral telencephalon and spinal cord, neuronal fates can be changed by interfering with Shh signalling even after the onset of fate specific TF expression (Xu et al., 2005; Xu et al., 2010; Dessaud et al., 2010). Following our hypothesis, it thus seems that the adult NSC, rather than inheriting regional information from the radial glia precursor, in fact inherits responsiveness to environmental factors that are continuously expressed during life and can be changed by disruption of homeostasis.

It is also possible that neuronal subtype instruction continues after the neuroblast leaves its original birthplace. It is true that once they enter the migratory chains, the neuroblasts that give rise to different $\mathrm{OB}$ neuronal types face the same environmental factors. However, they behave differently there. First, there is a decreasing gradient of proliferation in the SVZ from posterior to anterior
(Coskun and Luskin, 2001). The different populations of neuroblasts also leave the cell cycle at different locations, as shown by the labelling of proliferative cells through retrovirus injection in the anterior SVZ (Luskin, 1993), and in the intersection between the descending and horizontal limbs of the rostral migratory stream (RMS) (Smith and Luskin, 1998; Suzuki and Goldman, 2003; Hack et al., 2005). Although labelled cells in the anterior SVZ produce mostly granular neurons $(65 \%$, compared with $25 \%$ found in the glomerular layer), $75 \%$ of the labelled cells after RMS injections go to the glomerular layer (Luskin, 1993; Smith and Luskin, 1998). These data indicate that different neuronal types have different timings of maturation. Although they migrate through the same migratory pathway and experience the same environmental signals, these signals can differentially affect them according to their history. In line with this idea, it is known that, in the developing cerebral cortex, cell responsiveness to environmental signals that regulate cortical layer identity depends on the phase of the cell cycle (McConnell and Kaznowski, 1991; see also Mizutani and Saito, 2005).

\section{Conclusions}

We propose here that progenitors of the SVZ are not terminally committed and instead are continuously sensing the environment in order to make decisions about their fate. Exposing these progenitors to new cues can lead to the generation of neuronal types that are not observed under normal physiological conditions, such as cortical pyramidal neurons (Magavi et al., 2000; Sequerra et al., 2010).

As an alternative to the existence of intrinsically restricted precursors, we hypothesize that SVZ progenitors possess the ability to give rise to a wide range of potential phenotypes but that these are normally hindered by intra- and extracellular signals. Therefore, general predictions about determinants of stem cell potential have to take into account a complex landscape that includes cell history (De Marchis et al., 2007) and changes in the environment (Magavi et al., 2000; Sequerra et al., 2010; see also Panchision and McKay, 2002).

In our view, the idea of differentiation as a unidirectional pathway is inconsistent with the data showing plasticity of progenitors that display lineage-specific TFs. The glutamatergic neuron-specific TF Neurog2 (Schuurmans et al., 2004), for example, shows oscillating expression in telencephalic progenitors that also oscillate the expression of Hes1 (Shimojo et al., 2008), a Notch signalling effector that inhibits neuronal differentiation (Bertrand et al., 2002; Kageyama et al., 2007). These oscillatory patterns open up the possibility that a progenitor that has experienced Neurog2 expression may switch to a different pathway during a phase of low expression (Fig. 4). Interesting data regarding how transcription profiles change during multipotent to lineage-specific transition have come from studies on haematopoietic cells. Pina et al. (Pina et al., 2012) show that early committed erythroid cells, analysed at the single cell level, are heterogeneous in their transcription profiles with characteristics that can include the expression of myeloid genes and the absence of typical erythroid genes. This variability is then resolved qualitatively and quantitatively during differentiation, but suggests that there are many molecular entry points for the same final result.

One prediction of this hypothesis is that intermediate progenitors generated from NSCs are instructed by environmental cues to follow differentiation into a specific neuronal type. Even after the onset of expression of type-specific TFs, intra- and extracellular signals would be required for the continuity of the differentiation pathway. Disruption of homeostasis or changes in extracellular signals could thus reroute these cells into a different pathway. This plasticity 
suggests that two neurons of the same type have possibly undergone different histories to reach a common result. Thus, although this hypothesis supports the instructive role of the positional information exerted on the primary precursor, it refutes the idea that this information is irrevocable and therefore not subject to plasticity.

Finally, the hypothesis finds resonance in the plausible supposition that a primordial stem cell in the dorsal telencephalon gives rise sequentially to glutamatergic neurons, embryonically, and GABAergic neurons, postnatally. Based on the discussion developed above, we have no reason to predict the existence of two separate progenitors in the SVZ that give rise to GABAergic and glutamatergic populations. The data presented here bring a new turn to the preponderating idea that TFs are the sole determinants of neurotransmitter phenotype determination and support the idea that micro-environmental changes found during the embryonicpostnatal-life transition are fundamental for directing SVZ cells to generate $\mathrm{OB}$ interneurons. When progenitors are challenged with cues from a different environment, the plasticity potential of these cells is revealed.

The description of mechanisms through which the environment regulates neuronal type choice opens up possibilities for understanding pathologies in which the balance between excitatory and inhibitory populations is disrupted, as is the case in developmental disorders, such as epilepsy and autism, and in brain lesions and neurodegeneration. Furthermore, this knowledge is essential for developing neuronal replacement strategies that use intrinsic progenitor pools or manipulated transplanted precursors.

\section{Funding}

E.B.S. is supported by Shriners Hospitals for Children postdoctoral fellowship. CNPq, FAPERN, CAPES and Alexander von Humbolt Foundation support work by M.R.C. Funding for J.R.L.M. comes from FINEP, FAPERJ and CNPq. Funding for C.H.-P. comes from Pronex-Faperi, CNPq, FAPERJ and CAPES. C.H.-P. and J.R.L.M. have common funding form IBN-Net and CNPq/PRONEX.

\section{Competing interests statement}

The authors declare no competing financial interests.

\section{References}

Akiba, Y., Sasaki, H., Huerta, P. T., Estevez, A. G., Baker, H. and Cave, J. W. (2009). gamma-Aminobutyric acid-mediated regulation of the activitydependent olfactory bulb dopaminergic phenotype. J. Neurosci. Res. 87, 2211 2221.

Altman, J. (1969). Autoradiographic and histological studies of postnatal neurogenesis. IV. Cell proliferation and migration in the anterior forebrain, with special reference to persisting neurogenesis in the olfactory bulb. J. Comp. Neurol. 137, 433-457.

Anderson, S. A., Eisenstat, D. D., Shi, L. and Rubenstein, J. L. (1997) Interneuron migration from basal forebrain to neocortex: dependence on Dlx genes. Science $\mathbf{2 7 8}, \mathbf{4 7 4 - 4 7 6}$

Batista-Brito, R., Close, J., Machold, R. and Fishell, G. (2008). The distinct temporal origins of olfactory bulb interneuron subtypes. J. Neurosci. $\mathbf{2 8}, \mathbf{3 9 6 6 -}$ 3975.

Bayer, S. A. (1983). 3H-thymidine-radiographic studies of neurogenesis in the rat olfactory bulb. Exp. Brain Res. 50, 329-340.

Bayer, S. A. (1985). Neuron production in the hippocampus and olfactory bulb of the adult rat brain: addition or replacement? Ann. New York Acad. Sci. 457 163-172.

Beckervordersandforth, R., Tripathi, P., Ninkovic, J., Bayam, E., Lepier, A. Stempfhuber, B., Kirchhoff, F., Hirrlinger, J., Haslinger, A., Lie, D. C. et al. (2010). In vivo fate mapping and expression analysis reveals molecular hallmarks of prospectively isolated adult neural stem cells. Cell Stem Cell 7, 744758.

Belgacem, Y. H. and Borodinsky, L. N. (2011). Sonic hedgehog signaling is decoded by calcium spike activity in the developing spinal cord. Proc. Natl. Acad. Sci. USA 108, 4482-4487.

Bertrand, N., Castro, D. S. and Guillemot, F. (2002). Proneural genes and the specification of neural cell types. Nat. Rev. Neurosci. 3, 517-530.

Betarbet, R., Zigova, T., Bakay, R. A. and Luskin, M. B. (1996). Dopaminergic and GABAergic interneurons of the olfactory bulb are derived from the neonatal subventricular zone. Int. J. Dev. Neurosci. 14, 921-930.
Bi, B., Salmaso, N., Komitova, M., Simonini, M. V., Silbereis, J., Cheng, E., Kim, J., Luft, S., Ment, L. R., Horvath, T. L. et al. (2011). Cortical glial fibrillary acidic protein-positive cells generate neurons after perinatal hypoxic injury. J. Neurosci. 31, 9205-9221.

Borodinsky, L. N., Root, C. M., Cronin, J. A., Sann, S. B., Gu, X. and Spitzer, N. C. (2004). Activity-dependent homeostatic specification of transmitter expression in embryonic neurons. Nature 429, 523-530.

Brill, M. S., Snapyan, M., Wohlfrom, H., Ninkovic, J., Jawerka, M., Mastick, G. S., Ashery-Padan, R., Saghatelyan, A., Berninger, B. and Götz, M. (2008). A dl×2- and pax6-dependent transcriptional code for periglomerular neuron specification in the adult olfactory bulb. J. Neurosci. 28, 6439-6452.

Brill, M. S., Ninkovic, J., Winpenny, E., Hodge, R. D., Ozen, I., Yang, R., Lepier, A., Gascón, S., Erdelyi, F., Szabo, G. et al. (2009). Adult generation of glutamatergic olfactory bulb interneurons. Nat. Neurosci. 12, 1524-1533.

Chen, J., Magavi, S. S. and Macklis, J. D. (2004). Neurogenesis of corticospinal motor neurons extending spinal projections in adult mice. Proc. Natl. Acad. Sci. USA 101, 16357-16362.

Cheng, L., Arata, A., Mizuguchi, R., Qian, Y., Karunaratne, A., Gray, P. A. Arata, S., Shirasawa, S., Bouchard, M., Luo, P. et al. (2004). Tlx3 and Tlx1 are post-mitotic selector genes determining glutamatergic over GABAergic cell fates. Nat. Neurosci. 7, 510-517

Cocas, L. A., Miyoshi, G., Carney, R. S., Sousa, V. H., Hirata, T., Jones, K. R., Fishell, G., Huntsman, M. M. and Corbin, J. G. (2009). Emx1-lineage progenitors differentially contribute to neural diversity in the striatum and amygdala. J. Neurosci. 29, 15933-15946.

Coskun, V. and Luskin, M. B. (2001). The expression pattern of the cell cycle inhibitor p19(INK4d) by progenitor cells of the rat embryonic telencephalon and neonatal anterior subventricular zone. J. Neurosci. 21, 3092-3103.

Costa, M. R., Kessaris, N., Richardson, W. D., Götz, M. and Hedin-Pereira, C. (2007). The marginal zone/layer I as a novel niche for neurogenesis and gliogenesis in developing cerebral cortex. J. Neurosci. 27, 11376-11388,

Costa, M. R., Götz, M. and Berninger, B. (2010). What determines neurogenic competence in glia? Brain Res. Rev. 63, 47-59.

Costa, M. R., Ortega, F., Brill, M. S., Beckervordersandforth, R., Petrone, C., Schroeder, T., Götz, M. and Berninger, B. (2011). Continuous live imaging of adult neural stem cell division and lineage progression in vitro. Development 138, 1057-1068.

De Marchis, S., Bovetti, S., Carletti, B., Hsieh, Y. C., Garzotto, D., Peretto, P., Fasolo, A., Puche, A. C. and Rossi, F. (2007). Generation of distinct types of periglomerular olfactory bulb interneurons during development and in adult mice: implication for intrinsic properties of the subventricular zone progenitor population. J. Neurosci. 27, 657-664.

Dessaud, E., Ribes, V., Balaskas, N., Yang, L. L., Pierani, A., Kicheva, A., Novitch, B. G., Briscoe, J. and Sasai, N. (2010). Dynamic assignment and maintenance of positional identity in the ventral neural tube by the morphogen sonic hedgehog. PLoS Biol. 8, e1000382.

Diaz, F., McKeehan, N., Kang, W. and Hébert, J. M. (2013). Apoptosis of glutamatergic neurons fails to trigger a neurogenic response in the adult neocortex. J. Neurosci. 33, 6278-6284.

Doetsch, F., García-Verdugo, J. M. and Alvarez-Buylla, A. (1997). Cellular composition and three-dimensional organization of the subventricular germinal zone in the adult mammalian brain. J. Neurosci. 17, 5046-5061.

Doetsch, F., Caillé, I., Lim, D. A., García-Verdugo, J. M. and Alvarez-Buylla, A. (1999). Subventricular zone astrocytes are neural stem cells in the adult mammalian brain. Cell 97, 703-716.

Doetsch, F., Petreanu, L., Caille, I., Garcia-Verdugo, J. M. and Alvarez-Buylla, A. (2002). EGF converts transit-amplifying neurogenic precursors in the adult brain into multipotent stem cells. Neuron 36, 1021-1034.

Fagel, D. M., Ganat, Y., Cheng, E., Silbereis, J., Ohkubo, Y., Ment, L. R. and Vaccarino, F. M. (2009). Fgfr1 is required for cortical regeneration and repair after perinatal hypoxia. J. Neurosci. 29, 1202-1211.

Fishell, G., Mason, C. A. and Hatten, M. E. (1993). Dispersion of neura progenitors within the germinal zones of the forebrain. Nature 362, 636-638.

Fogarty, M., Grist, M., Gelman, D., Marín, O., Pachnis, V. and Kessaris, N. (2007). Spatial genetic patterning of the embryonic neuroepithelium generates GABAergic interneuron diversity in the adult cortex. J. Neurosci. 27 10935-10946.

Gabay, L., Lowell, S., Rubin, L. L. and Anderson, D. J. (2003). Deregulation of dorsoventral patterning by FGF confers trilineage differentiation capacity on CNS stem cells in vitro. Neuron 40, 485-499.

Garcia, A. D., Doan, N. B., Imura, T., Bush, T. G. and Sofroniew, M. V. (2004). GFAP-expressing progenitors are the principal source of constitutive neurogenesis in adult mouse forebrain. Nat. Neurosci. 7, 1233-1241.

Gorski, J. A., Talley, T., Qiu, M., Puelles, L., Rubenstein, J. L. and Jones, K. R. (2002). Cortical excitatory neurons and glia, but not GABAergic neurons, are produced in the Emx1-expressing lineage. J. Neurosci. 22, 6309-6314

Götz, M. and Bolz, J. (1994). Differentiation of transmitter phenotypes in rat cerebral cortex. Eur. J. Neurosci. 6, 18-32. 
Gulacsi, A. and Lillien, L. (2003). Sonic hedgehog and bone morphogenetic protein regulate interneuron development from dorsal telencephalic progenitors in vitro. J. Neurosci. 23, 9862-9872.

Gunhaga, L., Jessell, T. M. and Edlund, T. (2000). Sonic hedgehog signaling at gastrula stages specifies ventral telencephalic cells in the chick embryo. Development 127, 3283-3293.

Gunhaga, L., Marklund, M., Sjödal, M., Hsieh, J. C., Jessell, T. M. and Edlund, T. (2003). Specification of dorsal telencephalic character by sequential Wnt and FGF signaling. Nat. Neurosci. 6, 701-707.

Guo, F., Maeda, Y., Ma, J., Xu, J., Horiuchi, M., Miers, L., Vaccarino, F. and Pleasure, D. (2010). Pyramidal neurons are generated from oligodendroglial progenitor cells in adult piriform cortex. J. Neurosci. 30, 12036-12049.

Hack, M. A., Saghatelyan, A., de Chevigny, A., Pfeifer, A., Ashery-Padan, R., Lledo, P. M. and Götz, M. (2005). Neuronal fate determinants of adult olfactory bulb neurogenesis. Nat. Neurosci. 8, 865-872.

He, W., Ingraham, C., Rising, L., Goderie, S. and Temple, S. (2001). Multipotent stem cells from the mouse basal forebrain contribute GABAergic neurons and oligodendrocytes to the cerebral cortex during embryogenesis. $J$ Neurosci. 21, 8854-8862

Hinds, J. W. (1968a). Autoradiographic study of histogenesis in the mouse olfactory bulb. I. Time of origin of neurons and neuroglia. J. Comp. Neurol. 134 287-304.

Hinds, J. W. (1968b). Autoradiographic study of histogenesis in the mouse olfactory bulb. II. Cell proliferation and migration. J. Comp. Neurol. 134, 305322

lacovitti, L., Lee, J., Joh, T. H. and Reis, D. J. (1987). Expression of tyrosine hydroxylase in neurons of cultured cerebral cortex: evidence for phenotypic plasticity in neurons of the CNS. J. Neurosci. 7. 1264-1270.

Ihrie, R. A., Shah, J. K., Harwell, C. C., Levine, J. H., Guinto, C. D., Lezameta, M., Kriegstein, A. R. and Alvarez-Buylla, A. (2011). Persistent sonic hedgehog signaling in adult brain determines neural stem cell positional identity. Neuron 71, 250-262

Imura, T., Kornblum, H. I. and Sofroniew, M. V. (2003). The predominant neural stem cell isolated from postnatal and adult forebrain but not early embryonic forebrain expresses GFAP. J. Neurosci. 23, 2824-2832.

Jakovcevski, I., Mayer, N. and Zecevic, N. (2011). Multiple origins of human neocortical interneurons are supported by distinct expression of transcription factors. Cereb. Cortex 21, 1771-1782.

Jessell, T. M. (2000). Neuronal specification in the spinal cord: inductive signals and transcriptional codes. Nat. Rev. Genet. 1, 20-29.

Kageyama, R., Ohtsuka, T. and Kobayashi, T. (2007). The Hes gene family: repressors and oscillators that orchestrate embryogenesis. Development 134 1243-1251.

Kelsch, W., Mosley, C. P., Lin, C. W. and Lois, C. (2007). Distinct mammalian precursors are committed to generate neurons with defined dendritic projection patterns. PLOS Biol. 5, e300.

Kohwi, M., Petryniak, M. A., Long, J. E., Ekker, M., Obata, K., Yanagawa, Y. Rubenstein, J. L. and Alvarez-Buylla, A. (2007). A subpopulation of olfactory bulb GABAergic interneurons is derived from Emx1- and Dlx5/6-expressing progenitors. J. Neurosci. 27, 6878-6891

Kosaka, T., Hataguchi, Y., Hama, K., Nagatsu, I. and Wu, J. Y. (1985) Coexistence of immunoreactivities for glutamate decarboxylase and tyrosine hydroxylase in some neurons in the periglomerular region of the rat main olfactory bulb: possible coexistence of gamma-aminobutyric acid (GABA) and dopamine. Brain Res. 343, 166-171.

Kriegstein, A. and Alvarez-Buylla, A. (2009). The glial nature of embryonic and adult neural stem cells. Annu. Rev. Neurosci. 32, 149-184.

Letinic, K., Zoncu, R. and Rakic, P. (2002). Origin of GABAergic neurons in the human neocortex. Nature 417, 645-649.

Lois, C., García-Verdugo, J. M. and Alvarez-Buylla, A. (1996). Chain migration of neuronal precursors. Science 271, 978-981.

Luskin, M. B. (1993). Restricted proliferation and migration of postnatally generated neurons derived from the forebrain subventricular zone. Neuron 11, 173-189.

Magavi, S. S., Leavitt, B. R. and Macklis, J. D. (2000). Induction of neurogenesis in the neocortex of adult mice. Nature 405, 951-955.

Marek, K. W., Kurtz, L. M. and Spitzer, N. C. (2010). cJun integrates calcium activity and tlx3 expression to regulate neurotransmitter specification. Nat. Neurosci. 13, 944-950.

McConnell, S. K. and Kaznowski, C. E. (1991). Cell cycle dependence of laminar determination in developing neocortex. Science 254, 282-285.

Menezes, J. R., Smith, C. M., Nelson, K. C. and Luskin, M. B. (1995). The division of neuronal progenitor cells during migration in the neonata mammalian forebrain. Mol. Cell. Neurosci. 6, 496-508.

Merkle, F. T., Mirzadeh, Z. and Alvarez-Buylla, A. (2007). Mosaic organization of neural stem cells in the adult brain. Science $\mathbf{3 1 7}, 381-384$

Mizutani, K. and Saito, T. (2005). Progenitors resume generating neurons after temporary inhibition of neurogenesis by Notch activation in the mammalian cerebral cortex. Development 132, 1295-1304.
Mori, K., Kishi, K. and Ojima, H. (1983). Distribution of dendrites of mitral, displaced mitral, tufted, and granule cells in the rabbit olfactory bulb. J. Comp. Neurol. 219, 339-355.

Morshead, C. M., Garcia, A. D., Sofroniew, M. V. and van Der Kooy, D. (2003). The ablation of glial fibrillary acidic protein-positive cells from the adult central nervous system results in the loss of forebrain neural stem cells but not retinal stem cells. Eur. J. Neurosci. 18, 76-84.

Ohira, K., Furuta, T., Hioki, H., Nakamura, K. C., Kuramoto, E., Tanaka, Y., Funatsu, N., Shimizu, K., Oishi, T., Hayashi, M. et al. (2010). Ischemiainduced neurogenesis of neocortical layer 1 progenitor cells. Nat. Neurosci. 13 173-179.

Orona, E., Scott, J. W. and Rainer, E. C. (1983). Different granule cell populations innervate superficial and deep regions of the external plexiform layer in rat olfactory bulb. J. Comp. Neurol. 217, 227-237.

Palmer, T. D., Markakis, E. A., Willhoite, A. R., Safar, F. and Gage, F. H. (1999) Fibroblast growth factor-2 activates a latent neurogenic program in neural stem cells from diverse regions of the adult CNS. J. Neurosci. 19, 8487-8497.

Panchision, D. M. and McKay, R. D. (2002). The control of neural stem cells by morphogenic signals. Curr. Opin. Genet. Dev. 12, 478-487.

Pastrana, E., Cheng, L. C. and Doetsch, F. (2009). Simultaneous prospective purification of adult subventricular zone neural stem cells and their progeny. Proc. Natl. Acad. Sci. USA 106, 6387-6392.

Pastrana, E., Silva-Vargas, V. and Doetsch, F. (2011). Eyes wide open: a critica review of sphere-formation as an assay for stem cells. Cell Stem Cell 8, 486-498.

Pina, C., Fugazza, C., Tipping, A. J., Brown, J., Soneji, S., Teles, J., Peterson, C. and Enver, T. (2012). Inferring rules of lineage commitment in haematopoiesis. Nat. Cell Biol. 14, 287-294.

Saha, B., Jaber, M. and Gaillard, A. (2012). Potentials of endogenous neural stem cells in cortical repair. Front. Cell. Neurosci. 6, 14

Schuurmans, C. and Guillemot, F. (2002). Molecular mechanisms underlying fate specification in the developing telencephalon. Curr. Opin. Neurobiol. 12, 26-34

Schuurmans, C., Armant, O., Nieto, M., Stenman, J. M., Britz, O., Klenin, N., Brown, C., Langevin, L. M., Seibt, J., Tang, H. et al. (2004). Sequential phases of cortical specification involve Neurogenin-dependent and independent pathways. EMBO J. 23, 2892-2902

Sequerra, E. B., Miyakoshi, L. M., Fróes, M. M., Menezes, J. R. and HedinPereira, C. (2010). Generation of glutamatergic neurons from postnatal and adult subventricular zone with pyramidal-like morphology. Cereb. Cortex $\mathbf{2 0}$ 2583-2591.

Shepherd, G. (1990). The Synaptic Organization of the Brain, 3rd edn. Oxford University Press.

Shimojo, H., Ohtsuka, T. and Kageyama, R. (2008). Oscillations in notch signaling regulate maintenance of neural progenitors. Neuron $\mathbf{5 8}, 52-64$.

Smith, C. M. and Luskin, M. B. (1998). Cell cycle length of olfactory bulb neuronal progenitors in the rostral migratory stream. Dev. Dyn. 213, 220-227.

Stenman, J., Toresson, H. and Campbell, K. (2003). Identification of two distinct progenitor populations in the lateral ganglionic eminence implications for striatal and olfactory bulb neurogenesis. J. Neurosci. 23, 167-174.

Suhonen, J. O., Peterson, D. A., Ray, J. and Gage, F. H. (1996). Differentiation of adult hippocampus-derived progenitors into olfactory neurons in vivo. Nature 383, 624-627.

Suzuki, S. O. and Goldman, J. E. (2003). Multiple cell populations in the early postnatal subventricular zone take distinct migratory pathways: a dynamic study of glial and neuronal progenitor migration. J. Neurosci. 23, 4240-4250.

Ventura, R. E. and Goldman, J. E. (2007). Dorsal radial glia generate olfactory bulb interneurons in the postnatal murine brain. J. Neurosci. 27, 4297-4302.

Vessal, M. and Darian-Smith, C. (2010). Adult neurogenesis occurs in primate sensorimotor cortex following cervical dorsal rhizotomy. J. Neurosci. 30, 86138623.

Willaime-Morawek, S., Seaberg, R. M., Batista, C., Labbé, E., Attisano, L., Gorski, J. A., Jones, K. R., Kam, A., Morshead, C. M. and van der Kooy, D. (2006). Embryonic cortical neural stem cells migrate ventrally and persist as postnatal striatal stem cells. J. Cell Biol. 175, 159-168.

Winpenny, E., Lebel-Potter, M., Fernandez, M. E., Brill, M. S., Götz, M. Guillemot, F. and Raineteau, O. (2011). Sequential generation of olfactory bulb glutamatergic neurons by Neurog2-expressing precursor cells. Neural Dev. 6, 12

Xu, Q., Wonders, C. P. and Anderson, S. A. (2005). Sonic hedgehog maintains the identity of cortical interneuron progenitors in the ventral telencephalon. Development 132, 4987-4998.

Xu, Q., Tam, M. and Anderson, S. A. (2008). Fate mapping Nkx2.1-lineage cells in the mouse telencephalon. J. Comp. Neurol. 506, 16-29.

Xu, Q., Guo, L., Moore, H., Waclaw, R. R., Campbell, K. and Anderson, S. A. (2010). Sonic hedgehog signaling confers ventral telencephalic progenitors with distinct cortical interneuron fates. Neuron 65, 328-340.

Young, K. M., Fogarty, M., Kessaris, N. and Richardson, W. D. (2007). Subventricular zone stem cells are heterogeneous with respect to their embryonic origins and neurogenic fates in the adult olfactory bulb. J. Neurosci. 27, 8286-8296. 